\title{
COMMUNICATIONS
}

\author{
ON TONOMETRIC VALUES \\ BY \\ Priestley Smith, \\ BIRMINGHAM.
}

IN a paper on the "Limitations of the Tonometer," published in $1915^{1}$; I ventured to point out that many users of the Schiötz tonometer were attributing to that excellent instrument a degree of precision in the measurement of intra-ocular pressure which its author had never claimed for it, and which no such instrument can ever.possibly achieve. I return to the subject for a different reason. A recent and important article by McClean, of New York ${ }^{2}$, throws much doubt on the indications of the Schiöt $z$ tonometer. My own experiments suggested that the Schiötz curve underestimates the intra-ocular pressure at ordinary levels by about $5 \mathrm{~mm}$. Hg.; Dr. McClean's go much further, for they appear to show that the instrument underestimates it at all levels and by much larger amounts. His tests were numerous and were evidently made with elaborate care, but it seems to me. that his results, as they stand, are not conclusive. They differ widely from those obtained by Prof. Schiötz, and from my own, and can hardly be accepted until the causes of the discrepancy are explained.

Principle of Eye-tonometry.-A tonometer, however perfect, cannot directly measure the intra-ocular pressure. It can only measure the impressibility of the eyeball, and this the Schiötz instrument, when skilfully used, does admirably. We infer the intra-ocular pressure from the impressibility of the eye, but the result involves uncertainty, for the degree of impressibility depends not only on the height of the internal pressure but also to some extent on the character of the eye-ball-on its extensibility, its pliability, its curvature, and so forth. Hence it happens-and this has been proved by experiment-that eyes giving the same tonometric readings may have different internal pressures,' and that eyes giving different readings may have the same internal pressure. The detailed tables originally given by Prof. Schiötz, and my own also, give ample proof that considerable errors must sometimes arise in this way. The following experiment of my own illustrates the point in an exaggerated way:

A pig's eye was tested with the Schiötz tonometer under three different conditions : first, immediately after its removal from a pig just killed; again after it had lain $3 \frac{1}{2}$ hours in normal saline with formalin 5 per cent.; and again after a further 20 hours in the same 
fluid. In each case the intraocular pressure, as established by a manometer, was $25 \mathrm{~mm}$. Hg.

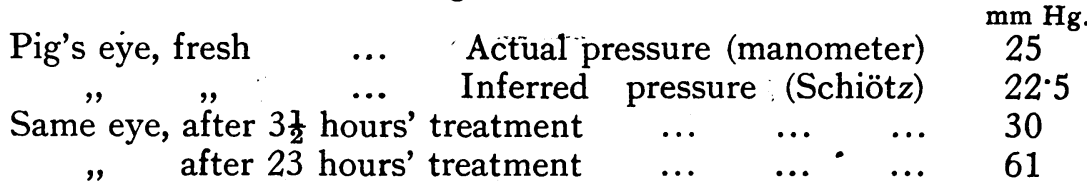

The formalin, of course, stiffened the tissues, diminished the impressibility of the eye, and falsified the mercury equivalents given by the Schiötz curve.

That tonometric measurements are inevitably beset by a margin of error-we do not yet know its width--is unfortunately often overlooked. Many suppose that a first-class instrument, well graduated and skilfully used, should measure the pressure in the eye as accurately as a thermometer measures the temperature of the blood. They forget that its action is indirect. Perhaps a rough illustration will be useful: The height of a child increases with its age, but the relation of height to age, among children, is not constant. We have tables, carefully prepared from numerous observations, that tell us the average relation, but if an enquirer, having provided himself with such a table, should attempt to ascertain the ages of individual children by measuring them, he would make some big mistakes, even though armed with the best measuring stick in the world.

Average equivalents of tonometer degrees, and how thev are obtained.-But though the degrees on the tonometer bear no constant relation to intra-ocular pressures, there is certainly an average relation, if it can be found ; each degree, on the average of many eyes, has an equivalent in $\mathrm{mm} . \mathrm{Hg}$. Such average equivalents can be presented in the form of a table or curve, or may be indicated on the scale of the instrument, and are valuable so long as it is remembered that in the case of any individual eye they may be incorrect by a good many $\mathrm{mm}$. $\mathrm{Hg}$. The difficulty is to determine them.

They are obtained by experimenting with the tonometer and a manometer simultaneously. The principle is simple enough, but its execution needs close attention to many details, and at the best it is impossible to make the tests under conditions precisely like those of the undisturbed living eye. Essentially the method is as follows:

The manometer, preferably measuring with water rather than mercury, is connected with an eye by means of a hollow needle, or canula, entering the vitreous chamber ; a known pressure is established in the eye; the connecting tube is then closed, and the tonometer is at once placed on the cornea ; its reading is recorded. This reading corresponds with that pressure-in that eye. In the same eye other pressures are then established, proceeding from the 
lower to the higher, and a reading is obtained for each. The whole proceeding is repeated on a number of eyes. Lastly, the average of the readings obtained for each pressure is found.

What the clinician wants to know, be it remembered, is the pressure which is present in the eye before the tonometer is placed ubon it, and it is the ralation of this to the scale of the instrument that the experimenter ascertains-as nearly as he can-by proceeding as above stated. Of course, the weight of the tonometer, when it is on a patient's eye, raises the intra-ocular pressure above its previous level, and the higher the previous level the more does it raise it, but that need not concern us, for it is the previous and not the modified pressure that we want to know.

Are the Schiötz curves correct ?-Prof. Schiötz obtained his data by experimenting, essentially as above described, on eight excised human eyes, and he published his results in detail. ${ }^{3}$ Seeing that the relation of the tonometer degrees to the intra-ocular pressures varied materially in different eyes, one may suppose that if more eyes had been tested the averages might have worked out a little differently. Also, seeing that these eyes were dead and that they were removed from their natural surroundings, one may doubt whether the findings are strictly applicable to living eyes. In view of these uncertainties, Prof. Schiötz expressly offered his figures as approximate equivalents, and in his subsequent tonometric work did not translate his findings into $\mathrm{mm}$. $\mathrm{Hg}$. . He is therefore not responsible if others have attached too precise a value to his curves.

My own experiments were undertaken to test the correctness of the Schiötz tonometer and its chart in the region of normal intra-ocular pressures. Until a few years ago the normal pressure was supposed to be about $25 \mathrm{~mm}$. $\mathrm{Hg}$. (Leber), ${ }^{4}$ this figure being in accordance with many manometric tests in animals, with a single such test in a healthy human eye, ${ }^{5}$ and with the average of many observations with the Maklakoff tonometer. ${ }^{6}$ The advent of "the Schiötz" made it possible to measure the impressibility of the eye with much greater precision than theretofore, and in course of time a careful observer, ${ }^{7}$ having found that 1,000 healthy eyes tested by himself gave an average reading of $4 \cdot 3$ degrees, declared the average pressure in healthy eyes to be $20 \mathrm{~mm}$. Hg., this being the Schiötz equivalent for that reading. But is the Schiötz curve correct ?

After some preparatory trials, I experimented in the way already described, first on eight fresh excised pigs' eyes, and then on twelve human eyes remaining in the orbit after death. In each case four different water-pressures were used. The tonometer had been tested by Prof. Schiötz. The findings were published in detail. ${ }^{1}$ In the following summary water-pressures are translated into their mercury equivalents : 


\begin{tabular}{|c|c|c|c|c|c|}
\hline ed & $\begin{array}{l}\text { Manometer } \\
\text { Schiötz tonometer }\end{array}$ & $\begin{array}{l}\text { mm. } \\
\mathrm{Hg} . \\
17{ }^{\circ} 6 \\
18\end{array}$ & $\begin{array}{c}\mathrm{mm} . \\
\mathrm{Hg} . \\
25 \\
25\end{array}$ & $\begin{array}{l}\mathrm{mm} . \\
\mathrm{Hg} . \\
32 \cdot 4 \\
32\end{array}$ & $\begin{array}{l}\mathrm{mm} . \\
\mathrm{Hg} . \\
39.7 \\
38.5\end{array}$ \\
\hline Huma & & 13 & 21 & $29 \cdot 5$ & 40 \\
\hline
\end{tabular}

It will be seen that the tonometer indicated the internal pressures in the pigs' eyes remarkably well, in other words that the Schiötz curve, orginally obtained by experimenting on excised human eyes, proved practically correct for excised pigs' eyes. On the other hand, it proved to be somewhat incorrect for human eyes that were not excised, especially with low pressures; with the 4 pressures the errors were $4 \cdot 5,4,2 \cdot 9$, and $0 \cdot 3 \mathrm{~mm}$. Hg., respectively. I think the discrepancy can be reasonably explained.

The eyes on which a tonometer is originally regulated (i.e., graduated, curves constructed, \&c.), should resemble those which it is subsequently to test, or its clinical indications are likely to be incorrect. The point is rather subtle but a little consideration will show that a tonometer which has been correctly regulated on eyes whose envelopes are of average extensibility will overstate the pressure in an eye whose envelope is relatively inextensible; while if it was regulated on eyes of less than average extensibility, it will understate the pressure in the average eye. Now there is reason to suppose that with equal internal pressures, and especially when these are low, excised eyes are somewhat less extensible than eyes remaining in the orbit. I quote the following from my earlier paper :

"In the orbit the sclera is to some extent supported from without by the soft cushion in which it lies, and is therefore less fully expanded by a given internal pressure than is the sclera of an excised eye; hence under the weight of the tonometer it is able to yield a little more - the fluid displaced by the tonometer is accommodated more easily, the impression is deeper. The difference will be more pronounced when the pressure is low in the eye than when it is high, for in the former case it will differ comparatively little from that of the orbital tissues and will be borne by them to a considerable extent, whereas in the latter it will be much the higher of the two and will be borne in larger proportion by the sclera itself. In an excised eye it is borne entirely by the sclera. This would explain the fact that the averages compared above differ less and less as the pressure rises."

If the foregoing explanation be true the small discrepancy between my own results and those of Professor Schiötz is explained, and it is probable that the Schiöt $z$ curve, in the neighbourhood of normal pressures puts the pressure lower than it really is. I suggest, moreover, that if we could accurately compare eyes remaining in the orbit after death with living eyes, this error in the Schiötz curve would prove to be somewhat greater than it here appears. 
Now McClean experimented not only on excised eyes, but also on living eyes of rabbits and dogs under ether, and on three living human eyes immediately before their removal for incurable glaucoma. $\mathrm{He}$ used the tonometer and manometer together in many cases. The results are interesting in many ways. Let us consider those bearing on the correctness of the Schiötz tonometer. It seems that in every case the tonometer put the intraocular pressure below its actual level, and as the discrepancies are too constant and too great to have arisen through exceptional peculiarities in the eyes examined, one can only hope to explain them by comparing McClean's material and methods with those which Schiötz employed in the regulation of his instrument, i.e., in constructing his curves. First as to the material.

McClean records five experiments on living rabbits, with nearly similar results, here is the first in brief :

Rabbit's eye, No. 1, cocaine, no ether. Schiötz tonometer $17 \cdot 5$

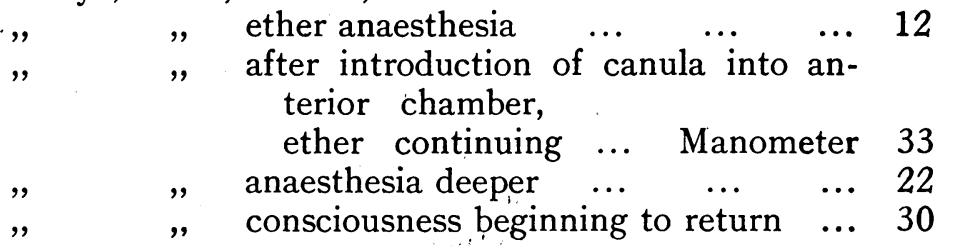

In this case, the manipulation on introducing the canula may perhaps have raised the intraocular pressure in some degree after the tonometric reading was taken, but one can hardly doubt that the original pressure was greater than shown by the tonometer. Yet we must not hastily blame the latter. The Schiötz tonometer was regulated on human eyes. The experiment does not show that it is untrue for human eyes: Until it has been proved that a man's eye and a rabbit's eye, with equal internal pressures, give equal tonometric readings, we must not expect any tonometer to be true for both. According to my own experiments, excised pigs' eyes do nearly resemble excised human eyes in their reaction to the tonometer, at least at moderate pressure-levels, but I know of no accurate comparative tests with regard to other animals.

Another of McClean's records relates to dogs' eyes and shows that in these also the Schiötz tonometer put the intraocular pressure a good deal lower than it really was. The same criticism applies: the tonometer was not regulated on dogs' eyes; though wrong for these it may be right for human eyes. Such evidence does not prove the instrument to be at fault.

The results of his experiments on human eyes are more disconcerting. He tested a Schiötz tonometer against a manometer on three living eyes blinded by glaucoma, under ether anaesthesia, immediately before their removal, and repeated the test on one of 
them after its removal. Here are some of the figures from the double test :

Human eye, living, glaucomatous, under ether. mm. Hg. mm. Hg.

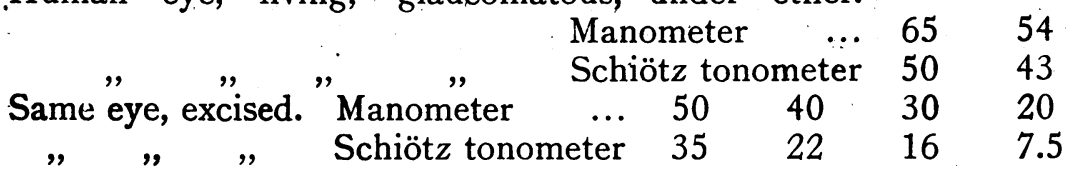

These are large discrepancies; what is the explanation? Is it that McClean tested the tonometer on living eyes whereas Schiötz regulated it on dead ones? No, for McClean tested it also on a living eye and it made the same mistakes. Is it that, with equal internal pressures, eyes blinded by glaucoma have less resilient envelopes than ordinary eyes artificially made tense? No, for the tonometer would relatively over-state the pressure in the stifferwalled eyes, and here it did the reverse.

Take a single one of these discrepancies and realise what it means. Schiötz established a pressure of $40 \mathrm{~mm}$. $\mathrm{Hg}$. in 8 eyes, applied his tonometer, noted the average position of the pointer, and made his chart agree therewith. I set up the same pressure in 12 eyes, applied a Schiöt $z$ tonometer, averaged the readings, and translated the result by the chart; it spoke correctly: the average reading indicated $40 \mathrm{~mm} . \mathrm{Hg}$. McClean set up this same pressure in the eye in question, applied the tonometer, and referred to the chart; it made a big mistake : it indicated $22 \mathrm{~mm}$. Hg. instead of 40 . Surely, his method of experimenting must havc differed in some essential way from that employed by Schiötz, and by myself. I will venture to suggest a possibility.

In many of his experiments McClean used a well-known method of control. A long horizontal glass tube, finely graduated and of small bore, is interposed between the manometer and the canula; an air bubble in the tube makes visible any movement of the water-column. When the bubble moves towards the manometer we know that the intraocular pressure is greater than the manometric, and that fluid is passing out of the eye through the canula ; and vice versa. Now, when the manometer is used for the purpose of measuring the pressure in a living eye this control is indispensable; the tube being open and the bubble stationary, the experimenter knows that the intraocular pressure is exactly equal to that indicated by the manometer. On the other hand, when the purpose is to test a tonometer against a known intra-ocular pressure, the tube must be closed before the tonometer is placed upon the eye, or the test will be vitiated.

Consider what happens if the tube remains open. The weight of the tonometer forces fluid out of the eye through the canula and drives the bubble backwards until the intraocular pressure is again 
equal to the manometric. The intraocular pressure therewith resumes its previous level, instead of remaining raised (modified) as in ordinary tests, for the amount of water driven out is too small to raise the water-level in the manometer in any discoverable degree. The tonometer, therefore, makes a larger and deeper pit in the cornea than in an ordinary test : the test is vitiated by the escape of fluid through the canula. It is true that the experimenter can prevent the escape, if he care to do so, by raising the manometric pressure sufficiently to hold the bubble in its original position. The manometer will then again measure the intra-ocular pressure. But what is it now measuring? Not the previous pressure, which is what he wants to know, but the modified pressure which is considerably higher, and with which he has no concern.

If McClean, in testing the Schiötz tonometer, left the tube open when the instrument was on the eye, and prevented displacement of the bubble by raising his manometric pressure, the discrepancies are to a large extent accounted for. If not, my suggestion fails, and another explanation must be sought. Unfortunately he leaves us in doubt on this crucial point. $\mathrm{He}$ speaks of using a stop-tap in one form of his apparatus, and of closing it before applying the tonometer, but with regard to the patency of the control-tube in the experiments here in question he says nothing. We want that information.

Summary.-The Schiötz tonometer measures the impressibility of the eye and can indicate changes of intra-ocular pressure in a given eye with a great certainty. For example, it has proved (see McClean's experiments) that in animals and in man, and that in glaucomatous as well as in healthy eyes, the intra-ocular pressure falls considerably during the introduction of ether anaesthesia.

The tonometer cannot measure the intra-ocular pressure with precision because the relation of the impressibility of the eye to the intra-ocular pressure is different in different eyes. In other words, exact mercury equivalents for the degrees indicated by the pointer cannot be given.

Approximate mercury equivalents are found by testing a number of human eyes with the tonometer and a manometer almost simultaneously. It is not possible, however, to carry out the test under conditions quite like those under which the tonometer is ordinarily used. Equivalents found by experimenting on animal eyes are likely to be incorrect for human eyes.

The value of any new tonometer will depend not merely on the excellence of its mechanism, but also, and essentially, on the tests by which it was regulated. Full details of the method and of the results should be given.

The Schiötz curves are not likely to be precisely correct, even for the average living eye, for the reasons just given. They are 
necessarily sometimes at fault for the individual eye. On the other hand, it is not proved, and it is not likely, that the instrument errs to the extent that McClean's experiments suggest.

\title{
REFERENCES
}

I. Smith, Priestley.-Ophthal. Rev., 1915, p. 65.

2. McClean, W.-Arch. of:Ophthal., I919, p. 23.

3. Schiötz.-Arch. f. Augenheilk., Vol. LXII, p. 317.

4. Leber, Th. - Graefe-Saemisch Handbuch, I903, Vol. II, part 2, p. 321.

5. Wahlford.-See Leber, as above.

6. Maklakoff.-See Leber, as above.

7. Cridland, B. -Brit. Jl. of Ophthal., I917, p. 352.

\section{HOMONYMOUS HEMIANOPIA OCCURRING IN A CASE OF MALIGNANT MALARIA}

BY

\author{
A. Leonard Weakley, M.B., B.S.Lond., F.R.C.S. Ed., \\ Captain R.A.M.C. \\ OPHTHALMIC SPECIALIST TO A GENERAL HOSPITAL, B.E.F.
}

THE following case is, I think, worthy of record, because I can find no previous mention of a similar one:

Lieut. W., aged 28, admitted into hospital suffering from malignant malaria; was sent to see me because of defective vision on June 6,1918 . He walked into the room and I noticed that he walked like a man who had very defective vision, holding his hands rather far stretched out and with his eyes widely open and staring. In answer to the question as to what he complained of, he said that his sight had gone; he could not see where he was going, and it was as though he were "shut in at the sides." He told me that he had come down the line with malaria and had had numerous injections of quinine en route. When I heard this I thought to myself at last here is a case of quinine amblyopia. (NOTE.-I have still to meet one here-after over three years of ophthalmic work and with thousands of cases of malaria and hundreds of thousands of men taking quinine, there has been no case of quinine amblyopia, and neither have I heard of one at any of the other hospitals.) However, on further questioning him he stated, with many pauses for recollection, that on May 23, when he got out of bed to wash, he suddenly felt as though he had been struck between the eyes and had a severe pain in the head right through to the back (occipital region), and found that he could not see properly and had not been able to since. $\mathrm{He}$ remembers nothing clearly from that time onwards until six days later on May 29, when he remembers being carried into hospital at Kantara, and has remembered things that happened after this. And from a statement by a friend who was in hospital with him he was "delirious" during this period. 